\title{
Bowenoid papulosis in a linear distribution
}

\author{
Wan-Su Peng, Cheng Tan
}

Department of Dermatology, Affiliated Hospital of Nanjing University of Chinese Medicine, Nanjing, China

Adv Dermatol Allergol 2016; XXXIII (2): 146-148

DOI: 10.5114/ada.2016.59161

We have recently encountered a bowenoid papulosis (BP) patient whose lesion distributed in a remarkable linear arrangement. Bowenoid papulosis was characterized by slightly elevated papules or plaques in the genital region that histologically resembled Bowen's disease (BD) [1]. Its lesions may be discrete or coalesce to form patches, rings to a rare linear patterns [2].

A 31-year-old male was referred to us for evaluation of a progressive, slow-growing lesion over the coronary sulcus of the penis. He had noticed its appearance 2 years before. The patient was otherwise in good health and denied any history of irradiation, trauma, or topical application of podophyllin. There was no antecedent infection of condyloma acuminate or herpes simplex. Skin examination revealed flat, elevated, well-demarcated, pigmented plaques on the coronary sulcus of his penis. These lesions were coalesced to an intermittent

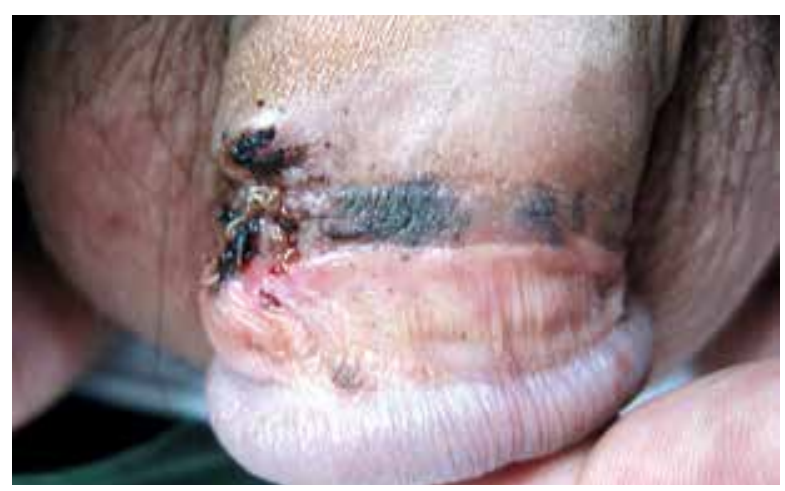

Figure 1. Flat, elevated, well-demarcated, pigmented plaques were noted on the coronary sulcus of his penis. These lesions were coalesced to an intermittent linear stripe that was vertical with the shaft of his penis. A few pin-sized similar smaller papules were scattered on the adjacent area linear stripe that was vertical with the shaft of his penis. A few pin-sized similar smaller papules were scattered on the adjacent area (Figure 1). Skin biopsy of the lesion revealed a hyperplastic epidermis with elongated epithelial ridges. There was full-thickness epidermal atypia with loss of orderly maturation, atypical mitosis, and dyskeratotic keratinocytes that were scattered throughout the epidermis. Within the dermis, there was a mild, superficial perivascular lymphocytic infiltrate with a few melanophages (Figure 2). On the basis of the clinical and histological features, the diagnosis of linear Bowenoid papulosis (LBP) was established. The affected area was ablated by a carbon dioxide laser and no relapse was observed after 2 years' follow-up.

Bowenoid papulosis was first described by Kopf and Bart in 1977 [3]. It predominantly affects individuals in their late third to mid-fourth decade of life. The duration of individual lesions ranges from a few weeks to over 10 years, with a median of 8 months. In men, the penile shaft, glans and foreskin are the most frequent sites of involvement, whereas in females, it primarily affects labia minor, labia major, clitoris, inguinal folds and perianal area $[1,4,5]$.

Bowenoid papulosis is commonly marked by macules (less frequent), papules or plaques which is usually less than $1 \mathrm{~cm}$ in size. The surfaces of the lesion mostly are flat, dome-shaped, papillomatous and verrucous. Bowenoid papulosis's colors are highly variable, they can be shiny flesh-colored, pink, reddish-brown, black, violaceous or may show coloration similar to that of the surrounding skin or mucosa [1, 2]. Most lesions of BP were described to be discrete, albeit some had an annular or linear exhibition. The latter condition is defined as LBP [4].

To the best of our knowledge, no more than 5 cases of LBP had been reported in the English literature prior to this [2, 4-7]. We found this subtype has following dis-

Address for correspondence: Cheng Tan MD, Department of Dermatology, Affiliated Hospital of Nanjing University of Chinese Medicine, 155 Hanzhong Road, 210029 Nanjing, China, phone: +86 13515102233, e-mail: tancheng@yeah.net, tancheng@medmail.com.cn Received: 13.04.2015, accepted: 30.05.2015. 

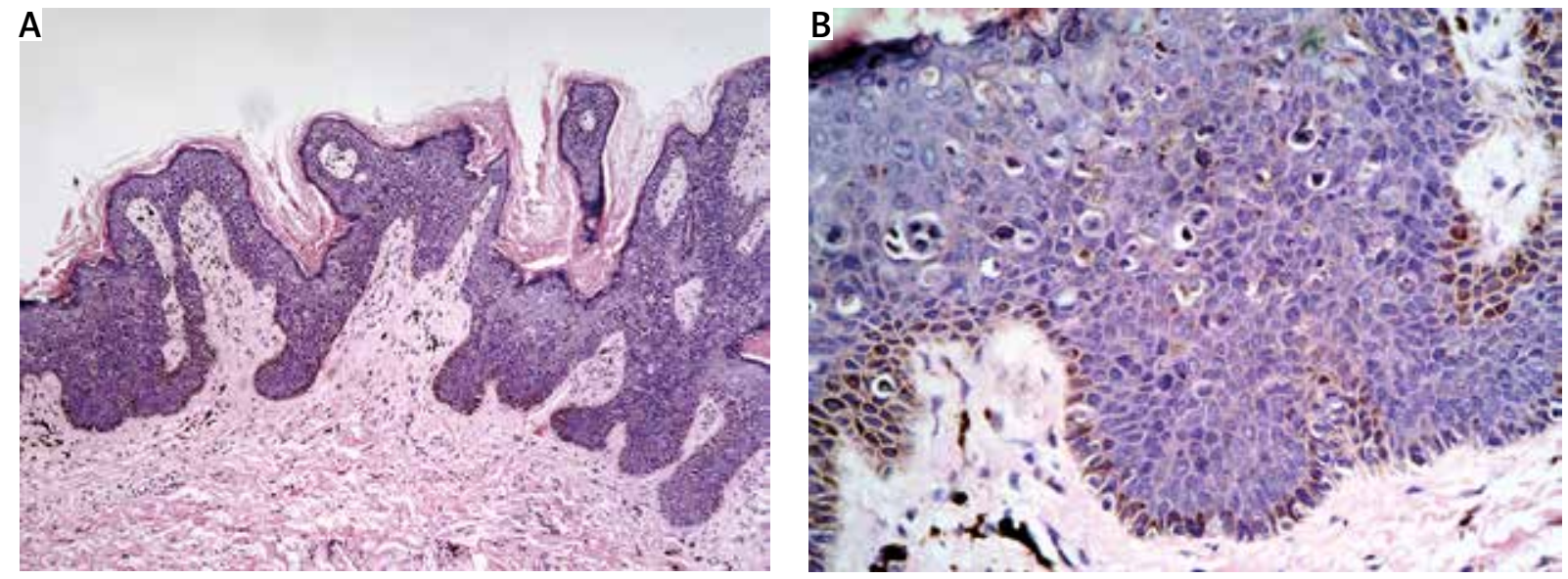

Figure 2. A - Microscopic examination disclosed epidermis with varying degrees of hyperkeratosis, irregular acanthosis, papillomatosis. Within the dermis, there was a mild, superficial perivascular lymphocytic infiltrate with a few melanophages (HE 100x). B - Higher magnification of A showing extreme nuclear atypia, dyskeratotic cells, and keratinocytes in mitosis at all levels of epidermis (HE 400x)

tinctive clinical features: 1) the lesions are intermittent or consecutive, linear configuration; 2) these linear lesions were distributed in a direction that is almost exclusively vertical with the shaft of the penis; 3 ) males are more susceptible than females ( 5 males out of 6 cases); 4) coronary sulcus and adjacent areas are the predominant sites of involvement.

Pathological changes of BP are very similar to BD. The epidermis was hyperplastic. There was full-thickness epidermal atypia with loss of orderly maturation, atypical mitosis, and dyskeratotic keratinocytes that were scattered throughout the epidermis. In the dermis, the vessels are dilated and tortuous, and there were scattered melanophages and a sparse perivascular infiltration of inflammatory cells [1].

Bowenoid papulosis is suggested by a lesion that clinically resembles a venereal wart but is reported as BD when biopsied. Bowenoid papulosis, BD and erythroplasia of Queyrat are distinct clinical entities with similar histological findings of intraepithelial neoplasia. Bowenoid papulosis may show acrotrichial sparing, less pronounced cellular dysplasia and mitotic figures, which helps its differentiation [1, 2]. Another histological differentiation of BP is Podophyllin treated condyloma accuminatum, which can be easily excluded by medical history of the patients.

Most cases of BP are due to high-risk human papillomavirus 16 (HPV-16), but in a small number, HPV-18, 31, $33,35,39$, and 53 , or mixed infections, have been present $[2,8]$. Some specialists advocate that LBP is a direct result of Koebner phenomenon [4] through direct contact or autoinoculation of these pathogens. Considering most lesions in LBP are distributed vertically with the shaft of the penis over the coronary sulcus, we deduced that, compared to other sites, HPVs residing in the coro- nary sulcus are more refractory to certain physiological elimination activities such as bathing or friction during intercourse possibly for its anatomical reasons. The possibilities of the development of LBP in this site might be greatly increased as a result of this prolonged retention of HPVs.

Bowenoid papulosis has a variable course, with a potential for regression, persistence, and recurrence. Treatment of BP usually involves locally destructive or ablative therapies such as excisional surgery, electrocoagulation, cryotherapy, and 5-fluorouracil. Several other treatments have also been reported, including topical 5-fluorouracil cream, $\mathrm{CO}_{2}$ laser therapy, podophyllin resin, imiquimod, electrocautery, photodynamic therapy or cryotherapy [1, $2,8]$.

\section{Acknowledgments}

The study was financially supported in part by the National Natural Science Foundation of China (Grant No. 81173400).

\section{Conflicts of interest}

The authors declare no conflict of interest.

\section{References}

1. Schwartz RA, Janniger CK. Bowenoid papulosis. J Am Acad Dermatol 1991; 24: 261-4.

2. Kumei A, Imanishi H, Tsuruta D, et al. Multiple linear bowenoid papulosis without surrounding papules. J Dermatol 2012; 39: 862-3.

3. Kopf AW, Bart RS. Tumor conference No. 11: multiple bowenoid papules of the penis: a new entity? I Dermatol Surg Oncol 1977; 3: 265-9. 
4. Sweidan NA, Salman SM, Zaynoun ST, et al. Linear bowenoid papulosis of the genitalia. A possible Koebner phenomenon. Int J Dermatol 1990; 29: 430-1.

5. Wade TR, Kopf AW, Ackerman AB. Bowenoid papulosis of the penis. Cancer 1978; 42: 1890-903.

6. Patterson JW, Kao GF, Graham JH, et al. Bowenoid papulosis. A clinicopathologic study with ultrastructural observations. Cancer 1986; 57: 823-36.

7. Berger BW, Hori Y. Multicentric bowen's disease of the genitalia: spontaneous regression of lesions. Arch Dermatol 1978; 114: 1698-9.

8. Pala S, Poleva I, Vocatura A. The presence of HPV types 6/11, $16 / 18,31 / 33 / 51$ in Bowenoid papulosis demonstrated by DNA in situ hybridization. Int J STD AIDS 2000; 11: 823-4. 